\title{
Critical Thinking for Critical Care
}

\author{
Leonard Azamfirei*
}

\section{Editor-in-Chief}

University of Medicine and Pharmacy of Tirgu Mures, 38 Gheorghe Marinescu street, Tirgu Mures, 540139, Romania

It is a great privilege to introduce the Journal of Critical Care Medicine, a new international publication aiming to promote the dissemination of scientific information in the treatment of the critical ill.

The JCCM, a multidisciplinary journal, will publish high-quality scientific papers covering different aspects related to the care of critical ill patients covering a broad spectrum of therapeutic disciplines, such as general surgery, perioperative care, cardiology, internal medicine, pneumology, infectious diseases, organ transplantation, emergency medicine or neurology.

Critical care medicine is a rapid expanding field. Recent progress in medical science and technology has led to a significant increase in the number of patients with life-threatening conditions who can benefit from increasingly wide-ranging monitoring and care. Significant resources are nowadays invested in the complex effort to discover and develop new approaches dedicated to improve the progress of these patients and to ensure a fast clinical and functional recovery.

In Central and Eastern European countries, complex strategies are required to align critical care models to the best current standards of care on international level. There is an obvious need, for a new journal dedicated to the exchange of information within the medical community dedicated to treating the critically ill. The necessity for a such a journal arises not only from the high relevance of the topics covered, but also from the paucity of journals in this part of Europe, dedicated to subjects relating to the critical ill. The JCCM will address these needs with specific regard, but not exclusively, to those of Central and Eastern European countries.

In the recently published WELPICUS study, it has been demonstrated that "World wide consensus could be developed for the majority of definitions and statements about end-of-life practices" and that these statements and definitions could be used in achieving universal standards of practice for end-of-life care. It was also suggested that statements where consensus was not reached, identified important areas for future research [1].

Integration, into their own sphere of influence, of worldwide accepted principles of care for the critical ill patient, represents a major objective for critical care specialists from Central and Eastern European. The JCCM will endeavor to provide the necessary scientific support to achieve this goal, concomitant with providing an efficient forum allowing the dissemination of knowledge and expertise together with the development of further research, in this key field of medicine.

One of the main goals of any medical journal is to contribute to the evidence base necessary to improve medical practice [2]. As a measure of social responsibility, the JCCM will stimulate publication of research that could be easily translated into current practice, and so doing will contribute to improved outcomes for critically-ill patients. Undoubtable, achieving these improvements represents an extremely difficult task. Not only does it entail a complex and multidisciplinary approach but also a high level of expertise in various different medical specialties. Covering different topics of medicine, ranging from pre-clinical to clinical studies and having the objective to publish the most recent advances in the field of research related to critical care medicine, the journal will promote the most up-to-date understanding of different pathophysiological mechanisms involved in the progression of disease together with the most contemporary accepted treatment of the critically ill. 
Academic criteria used for assessment of journal quality often fail to ensure a fair evaluation system, based as they are on citation levels, which do not always correlate with knowledge translation. A rapid social media recirculation via links to articles, added in rapidly spreading social communication channels, has the potential to change clinical practice, presenting sometimes a more significant impact than an article published in a top-ranking journal [3]. Some journals display on their web pages the "most downloaded articles" suggesting that the download frequency represents an alternative measure of article quality, independent of citation metrics. However, in the absence of alternative evaluation standards, the currently accepted academic criteria using citation analysis remains the basis of evaluation of journal content. The JCCM rapidly intends to reach a high citation score by selecting for publication articles of the highest quality. The diversity of published article types, ranging from "letter to editor" and "case presentation study" through "reviews" and "commissioned state-of-the-art articles" to the most complex original research papers, will ensure various possibilities for knowledge dissemination in the JCCM, and hopefully be reflected in the journal rapidly achieving a high citation record.
By launching the JCCM, we count on the contribution of the scientific community active in the field of critical care and we would like to encourage everyone concerned to use this opportunity and so make a significant contribution to the future development of critical care medicine.

We invite you to publish relevant papers in the JCCM, to enjoy together the growth of the journal and to share with us the success of this top-level academic initiative.

\section{REFERENCES}

1. Sprung $C L$, Truog RD, Curtis JR, et al. Seeking worldwide professional consensus on the principles of end-of-life care for the critically ill. The Consensus for Worldwide End-of-Life Practice for Patients in Intensive Care Units (WELPICUS) study. Am J Respir Crit Care Med. 2014;190:855-66.

2. Munro $\mathrm{CL}$, Savels RH. Stepping forward in practice through research. Am J Crit Care. 2014;23:4-7.

3. Weingart SD, Faust JS. Future evolution of traditional journals and social media medical education. Emerg Med Australas. 2014;26:62-6. 